\title{
A Contribution to Analysis of Collapse of High-Rise Building Inspired by the Collapses of WTC1 and WTC2: Derivation of Simple Formulas for Collapse Upper Speed and Acceleration
}

\author{
Ivan Němec1, Miroslav Trcala², Jiří Vala1, Adéla Vaněčková1 \\ ${ }^{1}$ Faculty of Civil Engineering, Brno University of Technology, Brno, Czech Republic \\ ${ }^{2}$ FEM Consulting, Brno, Czech Republic \\ Email: nemec@fem.cz
}

How to cite this paper: Němec, I., Trcala, M., Vala, J. and Vaněčková, A. (2018) A Contribution to Analysis of Collapse of High-Rise Building Inspired by the Collapses of WTC1 and WTC2: Derivation of Simple Formulas for Collapse Upper Speed and Acceleration. Journal of Applied Mathematics and Physics, 6, 2666-2680.

https://doi.org/10.4236/jamp.2018.612221

Received: November 27, 2018

Accepted: December 24, 2018

Published: December 27, 2018

Copyright $\odot 2018$ by authors and Scientific Research Publishing Inc. This work is licensed under the Creative Commons Attribution International License (CC BY 4.0).

http://creativecommons.org/licenses/by/4.0/

\begin{abstract}
The paper is a contribution to the technical discussion concerning the collapses of the WTC buildings. It returns to the problem of the dynamics of the collapses; it does not concern the reason why the buildings started collapsing, but investigates the dynamics of the collapse itself. It works with the same assumptions as the official NIST report [1], i.e. that the falling mass hits the motionless mass beneath; the supporting columns loose stability and the mass of the pertinent floor starts to fall together with the falling mass. The aim was to derive the theoretical upper limit of the speed of the collapse, supposing that influence of the columns which resist the fall, is neglected. The differential equation of the fall was obtained using two independent laws of mechanics, with the identical result. Its solution can be found from a very simple explicit formula. The theoretical upper limit acceleration of the fall obtained by such formula is one third of the gravitational acceleration, which is faster than it was observed in the case of the collapses of WTC1 and WTC2. This leads to the conclusion that the mechanism of the collapse must be different from the assumed and the falling mass must not hit the motionless mass bellow it, but rather a mass which had started to fall before the impact of the falling mass occurred.
\end{abstract}

\section{Keywords}

Progressive Collapse, WTC, High-Rise Building, Equation of Motion, Upper Limit Acceleration, 9/11 


\section{Introduction}

The fall of the WTC twin towers became the subject of a series of expert discussions on the mechanism of the fall and especially its speed. The official "final report" of National Institute of Standards and Technology (NIST) [1] states: "The release of potential energy due to downward movement of building mass above the buckled columns exceeded the strain energy that could be absorbed by the structure. Global collapse ensured". But how the downfall itself is then supposed to occur? What is the acceleration? Is the fall of the upper floors supposed to accelerate or should it decelerate and subsequently stop due to the resistance provided by the columns and other factors? These are issues that the NIST report does not deal with but which are dealt within the book [2] and this paper, inspired, indeed, by the fall of the twin towers, is devoted to the dynamics of the collapse of a high-rise buildings in general. In line with the conclusions of the NIST report, the authors assume that the fall has been initiated by the loss of stability of the columns in one or more floors of the high-rise building due to fire or any other starter.

The research of mechanics of the WTC twin towers' collapse has its own more 17 years old history: immediately (on September 13, 2001) Z. Bažant circulated his draft paper with results of a simple analysis of the WTC collapse: his suggestion was that heat from the fires was a key factor, causing steel columns in both the core and the perimeter to weaken and experience deformation before losing their carrying capacity and buckling; once more than half of the columns on a particular floor buckled, the overhead structure could no longer be supported and complete collapse of the structures occurred. The extended version of this analysis was then published in [3] and later in the substantially revised form in [4], whose differential equation of collapse of high-rise building involving various influences is based on the law of conservation of energy, as well as another comparable analysis [5], working with discrete series. Namely [4] notices that for some combination of parameters the fall would arrest, but does not present numerical solutions for such various combinations.

Several more official early reports were published namely by American Society of Civil Engineers, American Institute of Steel Constructions (AMSE), American Concrete Institute, National Fire Protection Association and Society of Fire Protection Engineers. The report of Federal Emergency Management Agency (FEMA) [6] suggested that fires in conjunction with damage resulting from the aircraft impacts were the key to the collapse of the towers. NIST conducted a 3-year, \$16 million investigation into the structural failure and progressive collapse of some WTC complex structures, with the output [1]. The scope of the NIST investigation was focused on identifying "the sequence of events" that triggered the collapse, using certain set of quasistatic models, but is not able to explain the collapse mechanism itself: the fires on particular floors, along with the lack of water, were also detected as the key reasons for the collapse. However, [7] concluded that such fires alone have been enough to bring down the buildings. Numerous publications like [8]-[14] oppose the official NIST explanation 
of the WTC collapse [1], pointing to the presence of thermitic material in the WTC dust, or to fundamental physics, but other ones, including all NIST official reports, defend it.

Later investigations work typically with advanced computational simulations, paying attention to various aspects of the fall of the WTC twin towers, as [15] studying the collapse behaviour of framed structures, [16] relying on the stochastic analysis, or [17] estimating the effect of temperature changes on mechanical properties of a model high-rise building; more extensive references can be found in [2]. The recent study [18] opens the possibility of damage modeling using a Gaussian springs based applied element method. Nevertheless, in the case of WTC collapse there is difficult to avoid setting many parameters, whose reliable values (or their probabilistic distributions) cannot be reconstructed from available sources like [1] and [6]. Such reasons are not dealt within this paper: it is concerned exclusively with the dynamics of the fall after it has, for any reason, already begun.

The dynamics of crash of a high-rise building is always a relatively complex problem. As with any mechanical problem, the solution must be based on application of the fundamental laws of mechanics, which are the law of conservation of mass, the law of conservation of energy and the law of conservation of momentum. These laws are unquestionable. There are also some other equations that are needed for the solution. It is primarily the definition of the properties of the continuum with which the calculation works, namely the rate of deformation and the relationship between the stress tensor and strain tensors, the so-called constitutive relationship. These equations, however, do not have the nature of physical laws; they are only our simplified model of nature. In the following considerations we shall demonstrate, under simplifying assumptions neglecting the resistance of columns, that it is possible to obtain simple formulae for upper limit of acceleration and velocity of a high-rise building fall.

\section{Simple Formulae for Collapse Upper Speed and Acceleration}

Let us demonstrate the use the above-mentioned basic laws of mechanics by a simple problem of a billiard ball moving at the speed hitting a stationary billiard ball. Let us assume that both balls have the same mass and the impact is centric.

Further, let us first assume that the balls are perfectly elastic, i.e. there occurs no dissipation of energy and plastic deformation during the crash. Then the law of conservation of momentum can be written in the form

$$
m v_{01}=m v_{1}+m v_{2}
$$

where $v_{1}$ and $v_{2}$ are the speeds of the two balls after the collision. Let us write the law of conservation of energy in a similar way. Given the zero dissipation, it only expresses the equality of the kinetic energy before and after the collision:

$$
\frac{1}{2} m v_{01}^{2}=\frac{1}{2} m v_{1}^{2}+\frac{1}{2} m v_{2}^{2} \text {. }
$$


The result of solution of this system of equations is that the moving ball stops after the collision (thus $V_{1}=0$ ) and the ball that was at rest before the collision will move at the speed $v_{01}$ after the collision $\left(v_{2}=v_{01}\right)$.

Now let us consider the case that the balls are not elastic, but perfectly plastic. This means that they will not rebound from each other upon the collision but they will move together, i.e. $v_{2}=v_{1}=v$. This particular option is of interest from the viewpoint of the fall of a high-rise building. Then an equation expressing the law of conservation of momentum can be written as follows:

$$
m v_{01}=m v_{1}+m v_{1} \Rightarrow v_{1}=v_{2}=v=v_{01} / 2
$$

The solution is thus obtained from a single equation and the result is that after the collision, both balls will move at half of the speed of the moving ball before the collision, as shown in Figure 1. When substituting into an expression for the kinetic energy, we can see that the kinetic energy after the collision will be only half of the kinetic energy before the collision. The remainder of the kinetic energy

$$
W_{p l}=\frac{1}{2} m v_{01}^{2}-\frac{1}{2} 2 m\left(\frac{v_{01}}{2}\right)^{2}=\frac{1}{4} m v_{01}^{2}
$$

is converted into heat, which is transferred into the free space (the so-called energy dissipation).

Let us now generalize the case of the perfectly plastic balls so that each ball is allowed to have a different mass. Let $m_{v}$ denote the mass of the ball moving before impact and $m_{s}$ the mass of the ball motionless before impact. Then the law of momentum conservation implies

$$
m_{v} v_{01}=\left(m_{v}+m_{s}\right) v \Rightarrow v=v_{01} \frac{m_{v}}{m_{v}+m_{s}}
$$

The kinetic energies before and after the impact can be express as follows:

$$
\begin{gathered}
E_{k 1}=\frac{1}{2} m_{v} v_{01}^{2}, \\
E_{k 2}=\frac{1}{2}\left(m_{v}+m_{s}\right) v^{2}=\frac{1}{2}\left(m_{v}+m_{s}\right)\left(v_{01} \frac{m_{v}}{m_{v}+m_{s}}\right)^{2}=\frac{v_{01}^{2}}{2} \frac{m_{v}^{2}}{m_{v}+m_{s}} .
\end{gathered}
$$

Consequently the dissipative energy can be expressed as the difference of the kinetic energy before and after the impact

$$
W_{p l}=E_{k 1}-E_{k 2}=\frac{1}{2} m_{v} v_{01}^{2}-\frac{v_{01}^{2}}{2} \frac{m_{v}^{2}}{m_{v}+m_{s}}\left(1-\frac{m_{v}}{m_{v}+m_{s}}\right)=\frac{1}{2} m_{v} v_{01}^{2} \frac{m_{s}}{m_{v}+m_{s}}
$$

The dynamics of the collapse of the high-rise building is a somewhat more

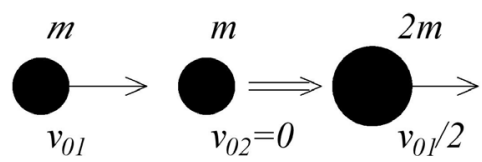

Figure 1. Collision of two plastic masses. 
complex problem but the theoretically possible limit speed of the fall can be determined by simple application of the law of conservation of energy, introducing considerably simplifying assumptions. Let us suppose that the mass of all the floors is the same and thus the mass is evenly distributed along the height of the building. The following solution is based on the assumption that the building begins to fall from the upper floors, which take down the lower floors and they then fall together. The progress of the collapse is shown in Figure 2.

When the front of the fall arrives to the place of coordinate, all the matter over this point will move with the same speed $v(x)$. This speed depends on many factors, but its upper limit may be estimated assuming that the columns do not impose any resistance to the fall and that all the falling material falls on the bottom floors, i.e. no material crashes out of the building. Neglecting the energy dissipation this upper speed limit can be evaluated from the equality of the potential energy $E_{p}$ of the building above the point $x$ related to the point and the kinetic energy $E_{k}$ in the moment of arrival of the front of the fall to the point $X$, therefore (Figure 3)

$$
\begin{gathered}
E_{p}=m(x) g \frac{x}{2}=\rho A x g \frac{x}{2}, \\
E_{k}=\frac{1}{2} m(x) v^{2}(x)=\frac{1}{2} \rho A x v^{2}(x)
\end{gathered}
$$

where $\rho$ is the average density of the building, $A$ is the area of the horizontal section of the building and $g$ is the local gravitational acceleration. If dissipation is neglected then the law of conservation of energy implies $E_{k}=E_{p}$.

Clearly (2) and (3) give

$$
\begin{aligned}
\frac{1}{2} \rho A x v^{2}(x) & =\rho A x g \frac{x}{2}, \\
v^{2}(x) & =g x
\end{aligned}
$$

and for the positive-valued $x$ and $v(x)$ just

$$
v(x)=\sqrt{g x}
$$

The speed of the matter falling freely from the height $x$ is

$$
v_{g}(x)=\sqrt{2 g x}
$$

The ratio of the limit speed to the speed of the free fall, or the inverse ratio of the corresponding times of the falls $T(x)$ and $T_{g}(x)$, can be therefore expressed as

$$
\frac{v(x)}{v_{g}(x)}=\frac{T_{g}(x)}{T(x)}=\frac{1}{\sqrt{2}}
$$

Let $a$ be the acceleration of the building collapse. For the ratio of the corresponding accelerations we have

$$
\frac{a}{g}=\frac{1}{2} .
$$

The velocity of the fall of a high-rise building, provided that the fall starts 


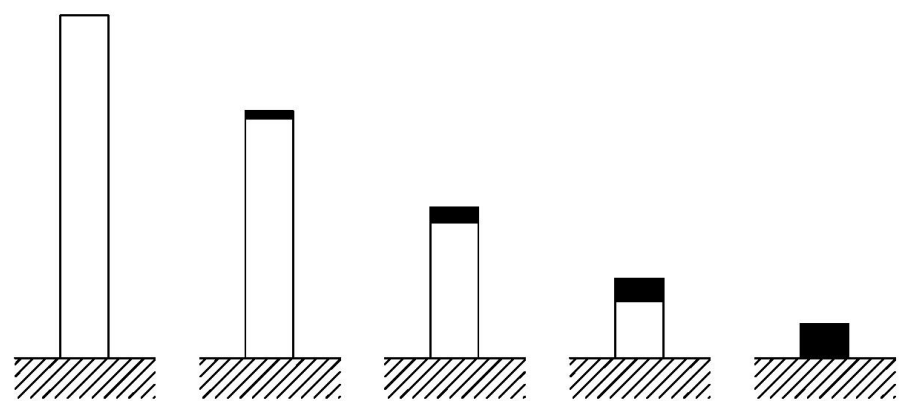

Figure 2. Phases of the progressive building collapse.

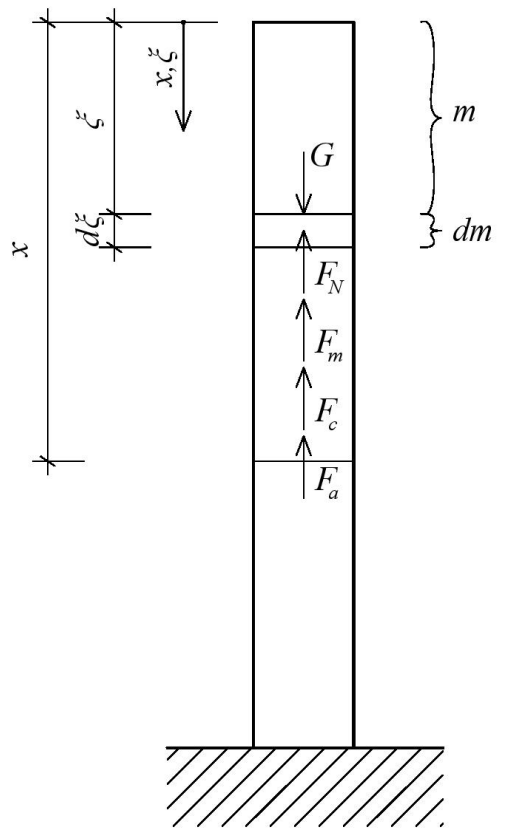

Figure 3. Scheme of a high-rise building.

from the top, can therefore be no more than $41 \%$ slower than the velocity of the free fall. The fall time will be at least $41 \%$ longer and the acceleration will be halved. Inclusion of the resistance imposed by the columns or any more accurate calculation (for example accounting for the fact that a part of the falling matter falls outside the building, and so does not hit the bottom of the building) can lead only to a slow-down in the fall or to its stop. Any plastic deformations of parts of a building lead to the dissipation of the energy, i.e. its conversion into thermal energy, which is, as it was seen before, at the expense of kinetic energy, i.e. in our case the speed of the fall. But the formulas velocity and acceleration introduced above were derived using the simplifying assumption when the dissipation energy is neglected. In the case of perfectly plastic balls, as described above, we have shown that the dissipation was substantial and should not be neglected. So let us include the energy dissipation into the problem of the progressive collapse of a high-rise building. Let us assume that the falling mass $m=\xi A \rho$ crash into motionless mass $\mathrm{d} m=\mathrm{d} \xi A \rho$ beneath and let us use for dissipative energy the Equation (1), where $m_{v}=m$ and $m_{s}=\mathrm{d} m$. The assumption 
that both masses move after the impact together, i.e. with the same speed, determine the dissipation energy regardless the magnitude of the strain and properties of its material uniquely. Neglecting the differential of mass $\mathrm{d} m$ in comparison to $m$ (i.e. assuming $m+\mathrm{d} m \approx m$ formally), we come to the formula for the differential increment of dissipation energy due to the impact

$$
\mathrm{d} W_{p l}(x)=\frac{1}{2} m v(x)^{2} \frac{\mathrm{d} m}{m+\mathrm{d} m}=\frac{1}{2} \mathrm{~d} m v(x)^{2}
$$

For the total amount of the dissipation energy from the beginning of the collapse until the mass reach the point $x$ we receive

$$
W_{p l}(x)=\int_{0}^{x} \mathrm{~d} W_{p l}(\xi)=\frac{1}{2} \int_{0}^{x} v^{2}(\xi) \mathrm{d} m=\frac{1}{2} \int_{0}^{x} v^{2}(\xi) A \rho \mathrm{d} \xi
$$

The kinetic energy of falling mass in the point $x$ can be then written in the form

$$
E_{k}(x)=E_{p}(x)-W_{p l}(x)=\frac{1}{2} m v^{2}(x)=\frac{1}{2} A \rho x v^{2}(x)
$$

where

$$
E_{p}(x)=m(x) g \frac{x}{2}=\operatorname{A\rho g} \frac{x^{2}}{2}
$$

is the potential energy of the building above the point $x$ to the point $x$. Inserting $E_{p}(x)$ from (6) and $x$ from (4) into (5), we obtain

$$
A \rho g \frac{x^{2}}{2}-\frac{1}{2} \int_{0}^{x} v^{2}(\xi) A \rho \mathrm{d} \xi=\frac{1}{2} A \rho x v^{2}(x)
$$

Consequently we have the resulting integral equation for the unknown function $v(x)$

$$
x v^{2}(x)+\int_{0}^{x} v^{2}(\xi) \mathrm{d} \xi=g x^{2} \text { for any } x \geq 0
$$

together with the initial condition

$$
v(0)=0
$$

and with the obvious requirement

$$
v(x)>0 \text { for any } x>0 \text {. }
$$

Let us differentiate (7) with respect to $x$ using the relation

$$
\frac{\mathrm{d}}{\mathrm{d} x}\left(\int_{0}^{x} v^{2}(\xi) \mathrm{d} \xi\right)=\frac{\mathrm{d}}{\mathrm{d} x}(F(x)-F(0))=\frac{\mathrm{d} F(x)}{\mathrm{d} x}=v^{2}(x)
$$

where $F($.) means a primitive function corresponding to $v 2($.$) . The obvious$ formal modification of (7) gives then the differential equation of collapse of a high-rise building, assuming that the columns do not resist the fall and all falling mass hits to the mass beneath, in the form, valid any for any $x>0$,

$$
\frac{\mathrm{d} v(x)}{\mathrm{d} x}=\frac{g x-v^{2}(x)}{v(x) x} .
$$


Clearly this differential equation can be rewritten as

$$
\begin{gathered}
v(x) \frac{\mathrm{d} v(x)}{\mathrm{d} x} x=g x-v(x)^{2} \\
v(x)\left(\frac{\mathrm{d} v(x)}{\mathrm{d} x} x+v(x)\right)=g x
\end{gathered}
$$

multiplying (11) by $x$ also as

$$
x v(x)\left(\frac{\mathrm{d} v(x)}{\mathrm{d} x} x+v(x)\right)=g x^{2}
$$

thus after integration, using some real constan $C$,

$$
\frac{1}{2}(x v(x))^{2}=\frac{g x^{3}}{3}+\frac{C}{2},
$$

which yields

$$
x v(x)= \pm \sqrt{\frac{2}{3} g x^{3}+C} .
$$

Then the solution of (9) can be expressed with respect to (8) in the explicit form

$$
v(x)=\frac{\sqrt{\frac{2}{3} g x^{3}+C}}{x}
$$

Moreover (7) forces $C=0$, which leads to the simple formula

$$
v(x)=\sqrt{\frac{2}{3} g x}
$$

for the evaluation of the required velocity $v(x)$.

Let us notice that the same result (12) can be also derived from the integral Equation (7) directly, taking $v(x)$ in the form $v^{2}(x)=c_{0}+c_{1} x$ with some real parameters $c 0$ and $c 1$. By (7) both these parameters must be non-negative, moreover (8) forces $c 0=0$. Then (7) yields $3 c 1 \times 2=2 g \times 2$, thus we obtain just the solution (12). However, we cannot be sure that some other solution does not exist. Therefore let consider be another hypothetical solution $w(x)$ of (7), (8) and (9), instead of $v(x)$. Introducing the notation $\varphi(x)=v^{2}(x)-w^{2}(x)$, from (6) we have

$$
\varphi(x)+\frac{1}{x} \int_{0}^{x} \varphi(\xi) \mathrm{d} \xi=0 \text { for any } x \geq 0,
$$

which is a very special Volterra equation: any non-zero value of $\varphi(x)$ should have an opposite sign than the mean value of the same function on the interval $(0, x)$, which leads to the contrary $\varphi(x)=0$ and $w(x)=v(x)$.

From comparison with velocity in free fall $v=\sqrt{2 g x}$ it follows that the acceleration of progressive collapse of a high-rise building in extreme, but theoretically possible case (i.e. if neither columns nor air resist the fall and all falling mass hits the building mass beneath) is only one $1 / 3$ of the acceleration of 
free fall,

$$
a=\frac{g}{3}
$$

Nevertheless, during the collapse of WCT1 and WCT2 the substantial portion of falling mass was observed not to hit the mass of the building beneath, but falling outside the building perimeter, thus there was no heap of ruins in the places where the buildings stood. Let us try to take into account also this influence and let us introduce the parameter $\beta$, which means the portion of the mass $\beta m$ hitting the motionless mass of the building beneath from the total falling mass. The remaining mass $(1-\beta) m$ falls aside and does not hit the building structure beneath.

We can again apply the law of conservation of energy in the form

$$
E_{p}=E_{k \beta}+E_{k(1-\beta)}+E_{p(1-\beta)}+W_{p l} .
$$

Here

$$
E_{p}=\int_{0}^{x} g \rho A \xi \mathrm{d} \xi=\frac{g \rho A x^{2}}{2}
$$

is the total potential energy of the building above the location $x$ falling to the location $x$,

$$
E_{k \beta}=\frac{1}{2} \beta \rho A x v^{2}(x)
$$

is the kinetic energy of the mass falling inside the building perimeter, moreover

$$
\mathrm{d} E_{k(1-\beta)}=\frac{1}{2}(1-\beta) \mathrm{d} m v^{2}(\xi)=\frac{1}{2}(1-\beta) \rho A \mathrm{~d} \xi v^{2}(\xi)
$$

is the kinetic energy of the mass element $(1-\beta) \mathrm{d} m=(1-\beta) \rho A \mathrm{~d} \xi$ falling outside the building perimeter in the location $(x-\xi)$, therefore

$$
E_{k(1-\beta)}=\int_{0}^{x} \mathrm{~d} E_{k(1-\beta)}=\int_{0}^{x} \frac{1-\beta}{2} \rho A v^{2}(\xi) \mathrm{d} \xi
$$

is the kinetic energy of the mass falling outside the building perimeter, and finally

$$
\mathrm{d} E_{p(1-\beta)}=(1-\beta) g \mathrm{~d} m(x-\xi)=(1-\beta) g \rho A(x-\xi) \mathrm{d} \xi
$$

is the potential energy of the mass element $(1-\beta) \mathrm{d} m=(1-\beta) \rho A \mathrm{~d} \xi$ falling outside the building perimeter from location $(x-\xi)$ to the location $x$, consequently

$$
E_{p(1-\beta)}=\int_{0}^{x} \mathrm{~d} E_{p(1-\beta)} \mathrm{d} \xi=\int_{0}^{x}(1-\beta) g \rho A(x-\xi) \mathrm{d} \xi=(1-\beta) g \rho A \frac{x^{2}}{2}
$$

is the potential energy of the mass falling outside the building perimeter to the location $x$.

For the plastic dissipation of energy we can write

$$
\begin{gathered}
\mathrm{d} W_{p l}=\frac{1}{2} \beta m v^{2} \frac{\mathrm{d} m}{\beta m}=\frac{1}{2} v^{2} \mathrm{~d} m, \\
W_{p l}=\frac{1}{2} \int_{0}^{x} \mathrm{~d} W_{p l}=\frac{1}{2} \int_{0}^{x} v^{2}(\xi) A \rho \mathrm{d} \xi .
\end{gathered}
$$


Then the law of conservation of energy reads

$$
\frac{g \rho A x^{2}}{2}=\frac{1}{2} \beta \rho A x v^{2}(x)+\int_{0}^{x} \frac{1-\beta}{2} \rho A v^{2}(\xi) \mathrm{d} \xi+(1-\beta) g \rho A \frac{x^{2}}{2}+\frac{1}{2} \int_{0}^{x} v^{2}(\xi) A \rho \mathrm{d} \xi
$$

After simple modifications we obtain the governing integral equation for the velocity in the shape

$$
\beta \frac{g x^{2}}{2}-\frac{1}{2} \beta x v^{2}(x)-\int_{0}^{x}\left(\frac{2-\beta}{2}\right) v^{2}(\xi) \mathrm{d} \xi=0
$$

together with the initial condition (8) and with the obvious requirement (9).

Let us differentiate (15) with respect to $x$ using the relation

$$
\frac{\mathrm{d}}{\mathrm{d} x}\left(\int_{0}^{x} v^{2}(\xi) \mathrm{d} \xi\right)=\frac{\mathrm{d}}{\mathrm{d} x}(F(x)-F(0))=\frac{\mathrm{d} F(x)}{\mathrm{d} x}=v^{2}(x)
$$

where $F($.) means a primitive function corresponding to $v 2($.$) . The formal$ modification of (15) gives then the differential equation of collapse of a high-rise building, assuming that the columns do not resist the fall, in the form, valid any for any $x>0$

$$
\frac{g \beta}{v(x)}-\frac{v(x)}{x}-\frac{\beta \mathrm{d} v(x)}{\mathrm{d} x}=0 .
$$

The analogy of (16) with (10) is evident from its simple modification

$$
\frac{\mathrm{d} v(x)}{d x}=\frac{g x-v^{2}(x)}{\beta v(x) x} .
$$

Consequently the same arguments, implementing the initial condition(8), the integration constant $C=0$, etc., including the notice related to direct integration, can be applied with the result

$$
v(x)=\sqrt{\frac{2 \beta g x}{2+\beta}} .
$$

The corresponding acceleration is then

$$
a=\frac{\beta g}{2+\beta} .
$$

Clearly for the particular case $\beta=1$ we come back to (12) and (13).

All formulae for the evaluation of velocity (17) and acceleration (18) have been derived using the law of conservation of energy. The same formulae can be derived also from another physical principle, the law of conservation of momentum. A very general equation of motion, what is the expression of the law of conservation of momentum, is introduced in [1]:

$$
G-F_{N}-F_{m}-F_{C}-F_{a}=0
$$

Here $H$ is the weight of a part of the building above the location of coordinate $x$, for which the equilibrium equation is formulated, $F_{N}$ is the resistance exerted by the columns against the collapse, $F_{m}$ is the resistance triggered by the impact of the falling part of the building into the motionless mass below it, $F_{C}$ is the viscous 
damping force and $F_{a}$ is the inertial force of the falling mass (Figure 3 ).

To obtain the upper limit of the velocity and acceleration, we omit the resistance of columns and the dynamic viscous damping and the simplified equation of motion then reads

$$
G-F_{m}-F_{a}=0
$$

where

$$
G=\beta m g=\beta \rho A x g .
$$

We can use the term $\alpha=v / \mathrm{d} t$ for acceleration of the infinitesimally small mass $\mathrm{d} m$ in the equation due to acceleration starting from zero up to the velocity. Introducing $v(x)=\mathrm{d} x / \mathrm{d} t$ again, we obtain

$$
\begin{gathered}
F_{m}=\mathrm{d} m \alpha=\mathrm{d} m \frac{v(x)}{\mathrm{d} t}=\mathrm{d} m \frac{v^{2}(x)}{\mathrm{d} x}=\rho A v^{2}(x), \\
F_{a}=\beta m \alpha=\beta m \frac{\mathrm{d} v(x)}{\mathrm{d} t}=\beta m v(x) \frac{\mathrm{d} v(x)}{\mathrm{d} x}=\beta \rho A x v \frac{\mathrm{d} v(x)}{\mathrm{d} x} .
\end{gathered}
$$

Substituting these expressions into (19) we obtain

$$
\rho A x g \beta-\rho A v^{2}(x)-\beta \rho A x v \frac{\mathrm{d} v(x)}{\mathrm{d} t}=x g \beta-v^{2}(x)-\beta x v(x) \frac{\mathrm{d} v(x)}{\mathrm{d} t}=0 .
$$

Dividing this equation by $v(x)$, the final form of the equation of motion derived from the law of momentum conservation is just (16). In other words: the same results can be derived from the energy and momentum conservation laws under certain physically transparent simplifying conditions.

Introducing, with regard to WTC1 a WTC2, a relatively conservative assumption that one half of the falling mass falls outside of the building perimeter $(\beta=$ $0.5)$, we obtain for velocity

$$
v(x)=\sqrt{\frac{g x}{2.5}} .
$$

Then the acceleration $a$, too, is only one fifth of the gravitational acceleration, i.e., unlike (13),

$$
a=\frac{g}{5}
$$

\section{Application to the Collapse of WTC1}

Let us apply the above derived theoretical limit speed on the collapse of the WTC1 tower, as reminded by Figure 4 and Figure 5. The height of the tower was $417 \mathrm{~m}$. The lowest limit of the time, applying the maximum theoretical limit of acceleration (1.35), is then

$$
t=\sqrt{\frac{2 h}{a}}=\sqrt{\frac{2 \times 417}{g / 3}}[\mathrm{~s}] \approx 15.82 \mathrm{~s}
$$

The time of the free fall would be 9.13 seconds. From the available videos it can be seen that the collapse of the WTC1 building was slightly slower than the 


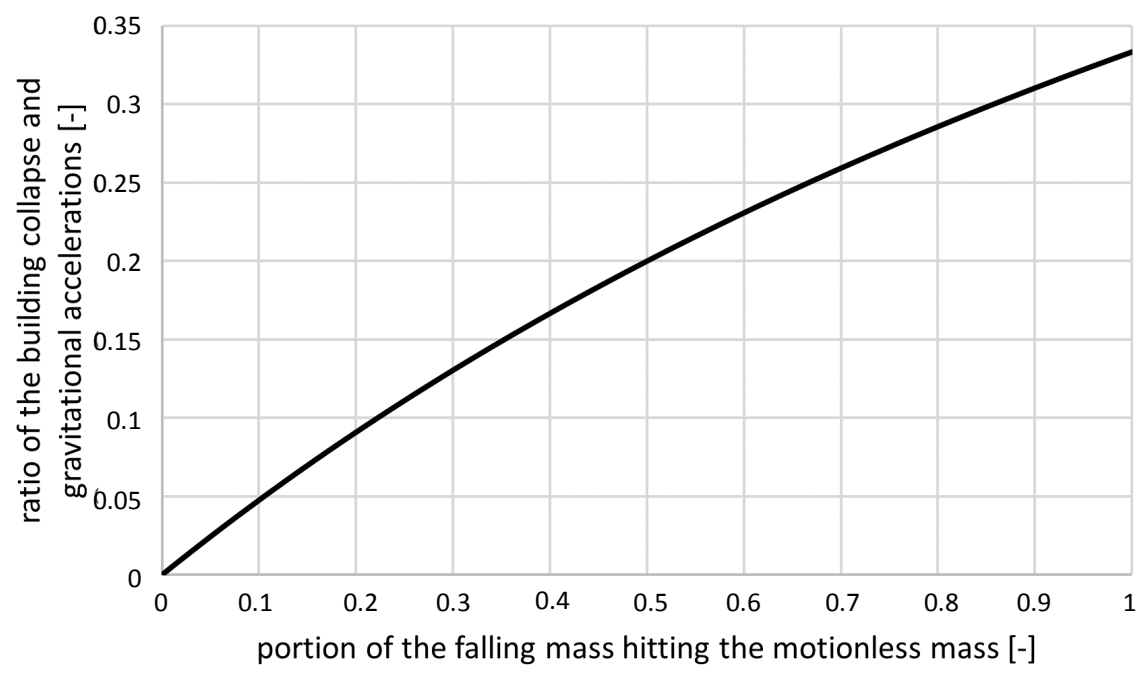

Figure 4. Resulting acceleration as a growing function of the parameter $\beta$ by (18): its maximum value for $\beta=1$ corresponds to (13).

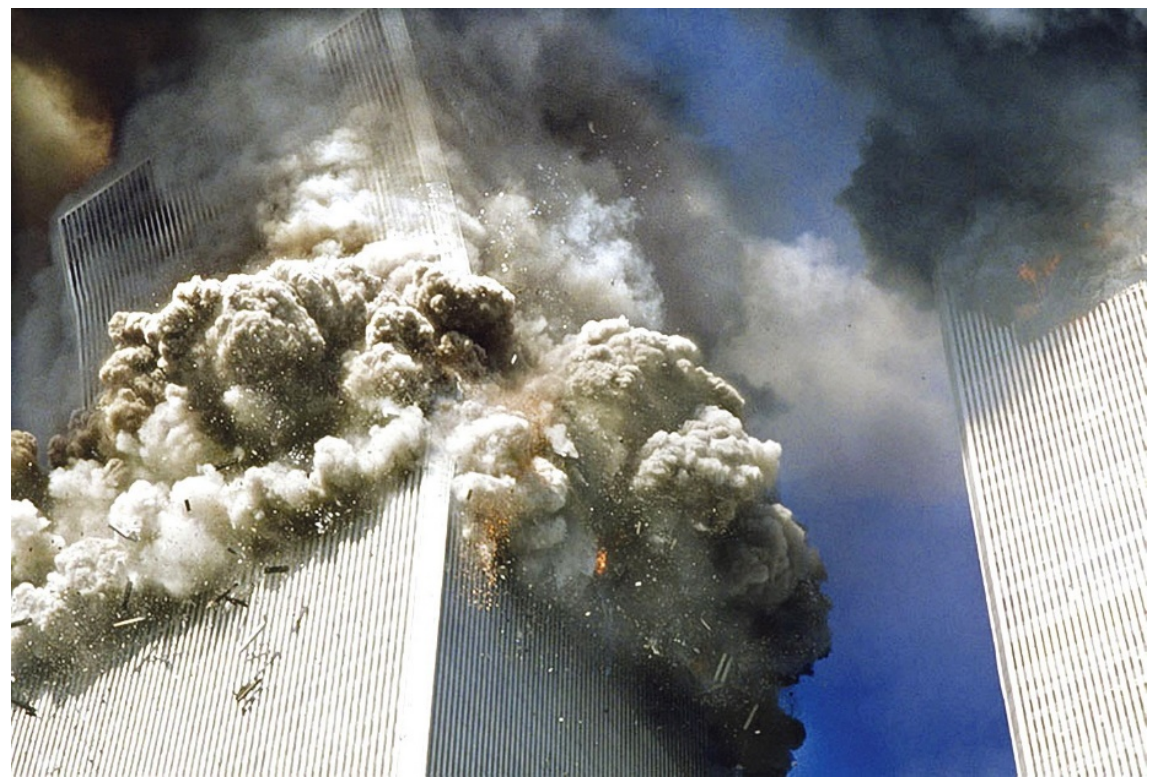

Figure 5. Documentary photo of the initial phase of the collapse of WTC2.

speed of free fall. We could thus estimate in concordance with many others that the collapse time of WTC1 was between 10 and 12 seconds. But by the upper limit acceleration formula, derived in this paper, we obtained the lowest limit collapse time 15.82 seconds. Even when we decrease the fall height by estimated $17 \mathrm{~m}$, due to the heap of ruins, we still obtain the theoretically least collapse time of 15.49 seconds, what is still higher than the observed collapse time between 10 and 12 seconds.

When taken into account the fact that a substantial part of the mass falls outside the perimeter of the building and does not hit the structure beneath, we obtain even bigger discrepancy between the theoretically upper limit of the speed of the collapse and the observed collapse time. Assuming that one half of the 
mass fallen outside the building perimeter $(\beta=0.5)$, which is, with regard to the videos and photos of the buildings site after the collapse, as illustrated by Figures 5-7, a rather conservative assumption, we obtain the time of collapse

$$
t=\sqrt{\frac{2 h}{a}}=\sqrt{\frac{2 \cdot 417}{g / 5}}[\mathrm{~s}] \approx 20.42 \mathrm{~s}
$$

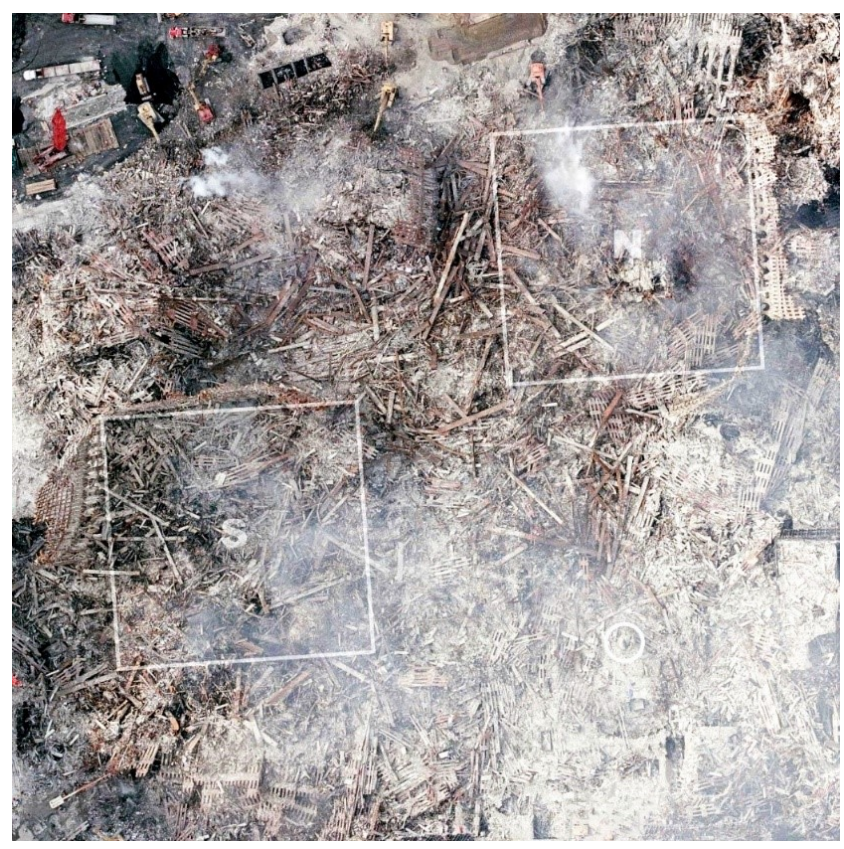

Figure 6. Photo of the situation just after the collapse of WTC1.

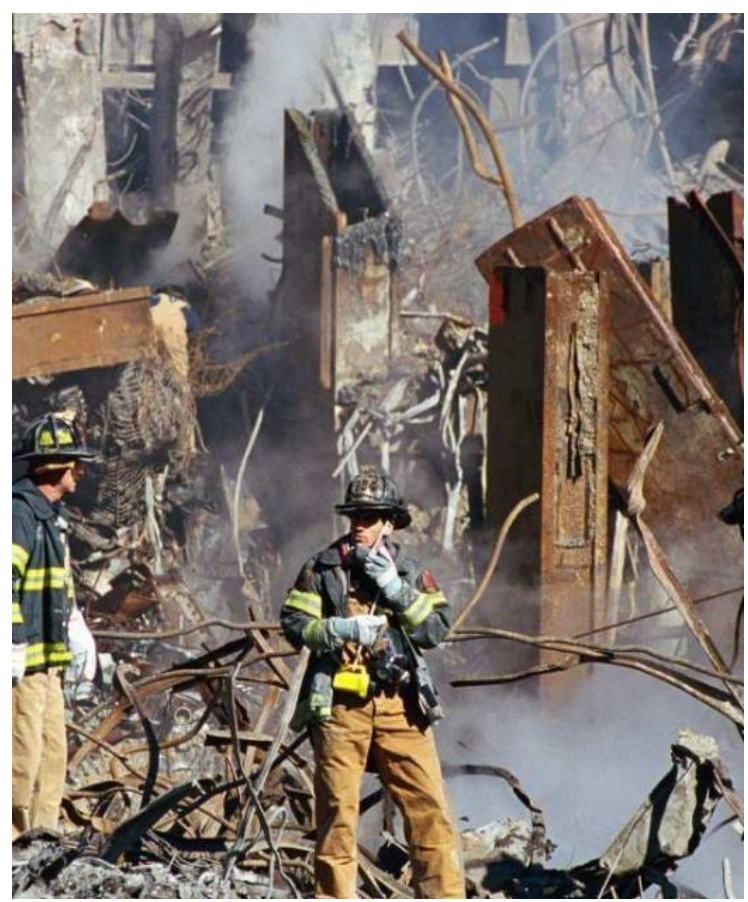

Figure 7. Probably the first research on the origin and mechanism of the collapse of WTC1. 
This is approximately twice of the observed time, and still no resistance of the columns was taken into account. It leads to the conclusion that the mechanics of the fall must be different than that supposed by the reports [1] and [5] and that the falling mass did not hit a motionless mass below it, but rather a mass which had started to fall before the impact of the falling mass occurred.

\section{Conclusions}

Let us emphasize that the simple formulas for acceleration of the fall of a high-rise building derived above have been obtained using the purely theoretical assumption that 1) columns are not resistible to the collapse, which defies their purpose, and that 2) there is also no resistance of air. However, this seems to correspond to real observations and is not in contradiction with the more detailed analysis of [2], as well as with some conclusions of [4] and [5].

Two different basic laws of mechanics give the same results concerning the upper theoretical limit of acceleration of the fall in an undisputable way, assuming the mechanism of the fall by the official NIST report [1], i.e. that the falling mass did hit a motionless mass below. The paper shows that the observed real collapses of the WTC1 and WTC2 were faster than such theoretical limit; thus the mechanism of the collapses must be different from that assumed in [1].

\section{Acknowledgements}

This study has been partially supported from the project of Ministry of Education, Youth and Sports of the Czech Republic AdMaS UP (Advanced Materials, Structures and Technologies), National Sustainability Programme I, LO1408.

\section{Conflicts of Interest}

The authors declare no conflicts of interest regarding the publication of this paper.

\section{References}

[1] NIST (2005) Final Report on the Collapse of the World Trade Center Towers. National Institute of Standards and Technology, Gaithersburg.

[2] Němec, I., et al. (2014) Dynamics of Collapse of a High-Rise Building. Scholars Press, Saarbrücken.

[3] Bažant, Z.P. and Zhou, Y. (2002) Why Did the World Trade Center Collapse?Simple analysis. Journal of Engineering Mechanics, 128, 2-6. https://doi.org/10.1061/(ASCE)0733-9399(2002)128:1(2)

[4] Bažant, Z.P. and Verdure, M. (2007) Mechanics of Progressive Collapse: Learning from World Trade Center and Building Demolitions. Journal of Engineering Mechanics, 133, 308-319. https://doi.org/10.1061/(ASCE)0733-9399(2007)133:3(308)

[5] Kuttler, K.L. (2006) A Short Computation. Journal of 9/11 Studies, 1-3.

[6] FEMA (2002) World Trade Center Building Performance Study: Data Collection, Preliminary Observations, and Recommendations. Federal Emergency Management Agency, Washington DC. 
[7] Usmani, A.S., Ghung, Y.C. and Torero, J.L. (2003) How Did the WTC Towers Collapse: A New Theory. Fire Safety Journal, 38, 501-533. https://doi.org/10.1016/S0379-7112(03)00069-9

[8] Jones, S. (2006) Why Indeed Did the WTC Buildings Completely Collapse? Journal of 9/11 Studies, 1-48.

[9] Harrit, N.H., et al. (2009) Active Thermitic Material Discovered in Dust from the 9/11 World Trade Center Catastrophe. The Open Chemical Physics Journal, 2, 7-31. https://doi.org/10.2174/1874412500902010007

[10] Szamboti, A. and MacQueen, G. (2009) The Missing Jolt: A Simple Refutation of the NIST-Bažant Collapse Hypothesis. Acta Radiologica, 42, 291-293.

[11] Chandler, D. (2010) The Destruction of the World Trade Center North Tower and Fundamental Physics. Journal of Studies, 1-17.

[12] Le, J.-L. and Bažant, Z.P. (2011) Why the Observed Motion History of World Trade Center Towers Is Smooth. Journal of Engineering Mechanics, 137, 82-84. https://doi.org/10.1061/(ASCE)EM.1943-7889.0000198

[13] MacQueen, G. (2013) Eyewitness Evidence of Explosions in the Twin Towers.

[14] Jones, S., Korol, R., Szamboti, A. and Walter, T. (2016) 15 Years Latter: On the Physics of High-Rise Building Collapses. Europhysics News, 447, 21-26. https://doi.org/10.1051/epn/2016402

[15] Isobe, D., Thanh, L.T.T. and Sasaki, Z. (2012) Numerical Simulation on the Collapse Behaviors of High-Rise Towers. International Journal of Protective Structures, 3, 1-20. https://doi.org/10.1260/2041-4196.3.1.1

[16] Irfanoglu, A. (2012) Using Numerical Simulations and Engineering Reasoning under Uncertainty: Studying the Collapse of WTC-1. Computer-Aided Civil and Infrastructure Engineering, 27, 65-76. https://doi.org/10.1111/j.1467-8667.2010.00700.x

[17] Zhu, K., Xu, K., Ansourian, P., Tahmasebibia, F. and Alonso-Marroquin, F. (2016) Energy Balance in the WTC Collapse. Numerical Methods of Civil Engineering: Dynamics of Structures, Proceedings of the Conference in Sydney, AIP Conference Proceedings, Vol. 1762, 1-16. https://doi.org/10.1063/1.4961105

[18] Abdul-Latif, M. and Feng, Y.T. (2018) Modelling Damage and Progressive Collapse of Frames Using a Gaussian Springs Based Applied Element Method. 6th European Conference on Computation-al Mechanics in Glasgow, 1-10. 